\title{
Prueba Argentina Psicolingüística de Denominación de Imágenes (PAPDI) Construcción y desarrollo (Primera parte)*
}

\section{Argentinean Psycholinguistic Image Naming Test (PAPDI). Design and development (First part)}

\author{
Laura Manoiloff**, Leticia Vivas***, Silvia Constanza Andreini****, \\ Nicolás Linares*****, Mercedes Soledad Fuentes Leiza******, Laura Del \\ Boca******* y Juan Segui $* * * * * * * *$
}

*Trabajo financiado por la Universidad Nacional de Córdoba (UNC), el Programa de Becas CarrilloOñativia y la Comisión Nacional Salud e Investigación del Ministerio de Salud de la Nación. **Doctora en Psicología. Directora del equipo de Investigación de Psicología Cognitiva del Lenguaje y Psicolingüística, Centro de Investigación de la Facultad de Psicología (CIPSI) Grupo Vinculado al Centro de Investigaciones y Estudios sobre Cultura y Sociedad (CIECS) - Consejo Nacional de Investigaciones Científicas y Técnicas (CONICET), Facultad de Psicología, Universidad Nacional de Córdoba (UNC). E-Mail: 1mvmanoiloff@gmail.com

Enrique Barros y Enfermera Gordillo s/n, Ciudad Universitaria. (5000) Córdoba, Argentina. ***Doctora en Neuropsicología Clínica. Miembro de la Carrera del Investigador Científico del Consejo Nacional de Investigaciones Científicas y Técnicas (CONICET) con funciones en el Instituto de Psicología Básica, Aplicada y Tecnología (IPSIBAT), Facultad de Psicología de la Universidad Nacional de Mar del Plata (UNMDP), CONICET.

****Licenciada en Psicología. Integrante del Laboratorio de Psicología Cognitiva, Centro de Investigación de la Facultad de Psicología (CIPSI), Grupo Vinculado al Centro de Investigaciones y Estu-

dios sobre Cultura y Sociedad (CIECS) - Consejo Nacional de Investigaciones Científicas y

Técnicas (CONICET), Universidad Nacional de Córdoba (UNC).

*****Médico. Médico de planta de la Sala de Neurología del Hospital Interzonal General de Agudos Oscar Alende. Mar del Plata.

******Licenciada en Psicología. Integrante del Laboratorio de Psicología Cognitiva del Centro de Investigación de la Facultad de Psicología (CIPSI), Grupo Vinculado al Centro de Investigaciones y Estudios sobre Cultura y Sociedad (CIECS) - Consejo Nacional de Investigaciones Científicas y

Técnicas (CONICET), Universidad Nacional de Córdoba (UNC).

*******Licenciada en Psicología. Co-directora del Equipo de Investigación de Psicología Cognitiva del Lenguaje y Psicolingüística. Laboratorio de Psicología Cognitiva del Centro de Investigación de la Facultad de Psicología (CIPSI), Grupo Vinculado al Centro de Investigaciones y Estudios sobre

Cultura y Sociedad (CIECS) - Consejo Nacional de Investigaciones Científicas y Técnicas (CONICET), Universidad Nacional de Córdoba (UNC).

********Doctor en Psicología Experimental. Director de Investigación Emérito del Centre National de la Recherche Scientifique (CNRS), Enseñante y Miembro del Consejo Pedagógico del Máster Europeo en Ciencias Cognitivas (COGMASTER) de Paris y Miembro del Laboratorio Mémoire et Cognition, Université, Paris, Descartes, Institut National de la Santé et de la Recherche Médicale (INSERM).

Facultad de Psicología de la Universidad Nacional de Córdoba (UNC), Instituto Privado de Neurociencias de Córdoba y Hospital Interzonal General de Agudos Oscar Alende (Mar del Plata). 


\section{Resumen}

En la primera parte de este trabajo se presenta la construcción y el desarrollo de la Prueba Argentina Psicolingüística de Denominación de Imágenes (PAPDI). La misma tiene dos características que la diferencian de las pruebas actualmente disponibles en nuestro medio: (a) Los estímulos fueron seleccionados controlando las variables que afectan las etapas de análisis visual $\mathrm{y}$ reconocimiento de la imagen (Complejidad visual y Concordancia con la imagen) y el conocimiento conceptual (Variabilidad de la Imagen y Familiaridad) del dibujo del objeto también se manipularon las variables (Frecuencia léxica y Edad de adquisición) que afectan la etapa que busca evaluar la prueba: activación, selección y recuperación de la etiqueta léxica. Los índices que operacionalizan a las variables son dependientes de la cultura y de la lengua de dónde se obtengan, por ello se tomaron sus valores de normas argentinas. (b) Las claves semánticas han sido elaboradas cuidadosamente, siguiendo las normas de producción de atributos semánticos también recolectadas en nuestro país, ya que el uso de las mismas permite generar hipótesis más precisas acerca de la localización del déficit. Por lo tanto, se presenta el proceso de construcción y desarrollo de la prueba mostrando cómo se tuvieron en cuenta estas particularidades, cuáles variables han sido controladas y cuáles manipuladas, y su justificación. Por último, los resultados de la prueba piloto permitieron seleccionar 30 ítems y la constitución de la versión final de la prueba, que es lo suficientemente breve para su uso en la clínica y de libre acceso.

Palabras clave: Prueba de Denominación de Imágenes; Construcción; Modelo de producción; Variables psicolingüísticas; Normas argentinas; Lenguaje.

\section{Abstract}

In the current paper, first part, we present the design and development of the Argentinean Psycholinguistic Image Naming Test (PAPDI). This test has two characteristics that make it different from those currently available in our country: (a) the stimuli have been selected by controlling the variables that affect the stages of visual analysis and image recognition (Visual Complexity and Image Agreement) and conceptual knowledge (Image Variability and Familiarity) of the drawing of the object; and the variables (Frequency of Use and Age of Acquisition) that affect the stage that seeks to evaluate the test were manipulated: activation, selection and retrieval of the lexical label (phonological form of the name), to generate a gradient of difficulty in the items of the test. However, the variable Naming Agreement, that also affects this stage, was controlled to selected pictures with only one predominating name. The quantification of these factors by means of specific variables is influenced by the cultural and linguistic context from which they were obtained, consequently we took their values from Argentinean normative data base and (b) semantic cues were elaborated according to semantic feature production norms also from Argentina. The phonological cues corresponded to the first syllable of the object's name. Their use allows to generate more precise hypotheses about the location of the deficit and allows to infer relevant information about the cognitive profile. Therefore, the process of construction and development of the test is presented, showing how these particularities were taken into consideration, which variables were controlled and which were manipulated, and their justification. For the design of the test 62 , black and white pictures were originally selected from the 400 pictures taken from Cycowicz's set (of frequent use in Experimental Psychology and Neuropsychology). They correspond to concrete concepts from different semantic categories belonging to both living and non living domains. The criteria used to include those images were that they were moderately complex (in quantity of lines and details), moderately familiar and that they had a mean image variability value. They had to have a medium to high concordance degree between the mental image and its corresponding representation. Besides, these images had to have a naming agreement superior to $80 \%$. To conform the first version of the test, the 62 images selected were ordered according to the variable Age of Acquisition and each image was assigned its corresponding 
semantic and phonological cues. The former were extracted from the Argentinean norms considering the most relevant features. The second consisted on the word's first syllable. A pilot study with healthy population $(n=50)$, of different ages, both sexes and three educational levels, was carried out through which 30 images were selected that constituted the test's final version. The deleted items were: figures that did not evoke a univocal response, that is, they were easily confused with other objects; figures that had more than one acceptable answer although not all were correct; and items that presented a ceiling effect where the scores accumulated in high scores and did not allow to discriminate the participants' ability to select, retrieve and produce a word (only 25 and $30 \%$ of the items were retained). In the test's final version, the items were reordered according to the Age of Acquisition and Frequency values and their difficulty in naming (following the percentage of successes in the spontaneous response). And images that were contain in another naming tests were deleted. The test is short enough for use in the clinic and freely accessible. The second part of the paper presents the psychometric studies that provide evidence of their relevance and validity.

Key words: Naming Test; Argentine norms; Design and development; Production model; Psycholinguistic variables; Language.

\section{Introducción}

La tarea de denominación de imágenes es de uso frecuente en la clínica neuropsicológica. Es un elemento indispensable en las baterías de evaluación de afasias, agnosias y distintos tipos de demencias, como Alzheimer o Afasia Progresiva Primaria entre otros (Cuetos, González \& De Vega, 2015; Grasso \& Peraita, 2011; Lezak, 1976; Martínez-Cuitiño \& Jaichenco, 2012; Moreno Martínez, 2006). Esta tarea implica varias etapas de procesamiento que pueden ser afectadas de manera diferente en los distintos síndromes neuropsicológicos. A su vez, cada etapa se ve influenciada por variables psicolingüísticas que pueden facilitar u obstaculizar la tarea. En general, los modelos (Glaser, 1992; Humphreys, Riddoch \& Quinlan, 1988; Levelt, Roelofs \& Meyer, 1999) acuerdan en una secuencia de cuatro etapas para dar cuenta del proceso de denominación y caracterizan las principales variables que afectan cada una de estas etapas. Dichas variables son caracterizadas y cuantificadas por índices específicos que sirven de predictores de la performance en la tarea de denominación (Alario et al., 2004; Barry, Morrison \& Ellis, 1997; Bonin, Boyer, Méot, Fayol \& Droit, 2004; Cuetos, Ellis \& Alvarez, 1999; Iyer, Saccuman, Bates \& Wulfeck, 2001).

La primera etapa de procesamiento corresponde al análisis y reconocimiento de la imagen. Esta etapa es afectada por factores como la Complejidad visual del objeto y la Concordancia con la imagen (por destacar el aspecto visuo-perceptivo del proceso). Por ejemplo, las imágenes con una alta complejidad visual son respondidas más lentamente que aquellas con una baja complejidad (Ellis \& Morrison, 1998). O las imágenes con alta concordancia entre la imagen mental del objeto y su representación pictórica son respondidas más rápidamente que las de baja concordancia (Barry et al., 1997).

En la siguiente etapa se accede al significado (concepto) que representa el dibujo, desde un almacén de conocimiento conceptual (para algunos autores, memoria semántica). Esta etapa se ve afectada por variables como la Variabilidad de la imagen y la Familiaridad, ya que son características directamente vinculadas con el conocimiento que tiene el sujeto sobre las propiedades del concepto al que se refiere la figura a denominar. Por ejemplo, las imágenes con gran variabilidad y que representan objetos más familiares son respondidas más rápido que las de baja variabilidad y poco familiares (Alario et al., 2004; Cuetos et al., 1999; Ellis \& Morrison, 1998; Snodgrass \& Yuditsky, 1996).

En una tercera etapa, la palabra que representa el concepto se activará en el léxico mental. Esta etapa corresponde a la activación, selección y recuperación de la forma fonológica del nombre (etiqueta léxica). Se ha demostrado que la Edad de adquisición (edad 
aproximada en la que se aprende la palabra), la Frecuencia léxica (frecuencia de uso en una lengua) y el Acuerdo en la palabra/s con que se denomina el objeto, son variables que influyen en el tiempo y exactitud de recuperación de la representación fonológica del nombre (Alario \& Ferrand, 1999; Alario et al., 2004; Barbón \& Cuetos, 2006; Bonin et al., 2004; Cuetos et al., 1999; Dell'Acqua, Lotto \& Job, 2000; Ellis \& Morrison, 1998; Jescheniak \& Levelt, 1994;,entre otros). La última etapa del procesamiento corresponde a la elaboración e implementación del programa articulatorio de la palabra seleccionada.

Como se puede vislumbrar en la descripción del modelo de procesamiento en la denominación de imágenes, se entrelazan dos tipos de códigos: uno no lingüístico (el pictorial) y otro lingüístico o lexical, más un almacén conceptual / semántico (Glaser, 1992) y estos se ven afectados diferencialmente por variables psicolingüísticas con distintas características. Sin embargo, el objetivo principal de esta tarea es evaluar la etapa de procesamiento de acceso, selección y recuperación del nombre del objeto. Si la denominación del objeto es efectuada correctamente, se infiere que todas las etapas previas de procesamiento se han efectuado adecuadamente. Si la persona falla en la denominación, queda por precisar cuál (o cuáles) etapas de procesamiento son afectadas.

En función de esto, hay dos puntos claves en la conformación de este tipo de pruebas. Primero, controlar las variables que afectan preferencialmente las diferentes etapas de procesamiento, al momento de seleccionar los estímulos; segundo, crear y controlar los facilitadores o ayudas para recuperar los nombres (dar la respuesta). Estos últimos se denominan claves y se clasifican en semánticas y fonológicas. Su uso permite generar hipótesis más precisas acerca de la localización del déficit.

En este trabajo se presenta el desarrollo de una prueba que fue diseñada controlando y manipulando los puntos esenciales arriba mencionados.

\section{Control de variables y claves}

En relación al control de las variables que afectan los diferentes niveles de procesamiento en la denominación, se cuenta actualmente con estudios normativos en sets de figuras experimentales para distintos idiomas. Snodgrass y Vanderwart (1980) fueron los primeros en elaborar un corpus de dibujos y obtener los valores de una muestra poblacional para las variables mencionadas. No obstante, estos datos son dependientes de la cultura y de la lengua de la muestra empleada. Por ejemplo, palabras que son frecuentes en un idioma, su traducción en otro idioma pueden corresponder a palabras poco frecuentes; o dentro de un mismo idioma como el español la palabra vale puede ser frecuente en el español peninsular y poco frecuente en el español rioplatense. Del mismo modo, un objeto pude ser denominado de forma unívoca en un idioma y poseer varios nombres en otro idioma. Esto llevó a realizar muchos estudios normativos sobre este set de imágenes en diferentes idiomas y culturas (Alario \& Ferrand, 1999; Dell'Acqua et al., 2000; Manoiloff et al., 2010; Martínez-Cuitiño, Barreyro, Wilson \& Jaichenco, 2015; Sanfeliu \& Fernández, 1996).

Dentro de estas variables, los índices de Acuerdo en el Nombre y la Frecuencia Léxica, son las variables más afectadas por el idioma y la cultura (Dell'Acqua et al., 2000; Manoiloff et al., 2010). Esta cuestión es particularmente importante para este trabajo dado que estas variables inciden directamente en la etapa que evalúa la prueba de denominación, por lo cual resulta fundamental el control y manipulación de las mismas.

Otro de los aspectos que tiene que ser fuertemente controlado en el diseño de una tarea de denominación es la selección de las claves. En la conformación de las claves semánticas, se suelen usar las categorías superordinadas y / o algún otro rasgo relevante. Por ejemplo, para abeja la clave sería es un insecto (categoría superordinada) o produce miel (rasgo relevante) o un insecto que produce miel (categoría superordinada y rasgo relevante), en el mejor de los casos. En pri- 
mer término, cuando al paciente se le presenta el dibujo, y no logra decir la palabra, entonces, se le da la clave semántica. Si responde adecuadamente, en principio, cabe suponer que no tiene afectada la representación semántica. O sea, o no reconocía el dibujo por una dificultad visuo perceptiva o no lograba discriminarlo de otros semejantes (en el caso de la abeja podría ser con una mosca). Al brindar la clave, pudo acceder al concepto, individualizarlo, así activar la palabra que representa a ese concepto (la representación de la forma fonológica del nombre) y lograr el nombramiento. Este resultado permitiría inferir que el contenido del almacén semántico se encuentra conservado (Jefferies, Patterson \& Lambon Ralph, 2008). Si el paciente reconoce el objeto y la categoría semántica a la que pertenece (la clave semántica no es de ayuda en este caso), pero no logra producir la palabra, entonces se proporciona la clave fonológica (que puede consistir en el primer fonema o sílabas del nombre del objeto). Si el paciente responde correctamente, esto indicaría que está afectada la vía de acceso léxico (no obstante, la información estaría conservada) y las etapas previas estarían conservadas.

La utilidad del uso de claves en los test de denominación se basa en el hecho de que los pacientes con afasia y demencia presentan distintos perfiles de respuesta. Hay trabajos que sugieren que las claves fonológicas son más efectivas que las semánticas en los pacientes con afasia fluente, mientras que las semánticas lo son en pacientes con Demencia tipo Alzheimer (Mehri, Jahani, Alemi \& Aramipour, 2008). A su vez, hay evidencia de que los pacientes con afasia post-ACV con presencia de déficits semánticos se benefician con la presentación de claves fonológicas, en oposición a los pacientes con Demencia Semántica quienes no suelen beneficiarse de ese recurso (Jefferies, Baker, Doran \& Lambon Ralph, 2007) indicando en este caso un deterioro en el contenido semántico. En el caso de los pacientes con Demencia Tipo Alzheimer se ha reportado que el beneficio que ofrecen las claves fonológicas disminuye a medida que avanza la enfermedad, sugiriendo que inicialmente habría una dificultad en el control ejecutivo del acceso a la información semántica pero que en etapas avanzadas se agregaría una progresiva degradación del repositorio semántico mismo con lo que las claves dejan de tener tal utilidad (Corbett, Jefferies, Burns \& Lambon Ralph, 2012).

\section{Pruebas de denominación disponibles en nuestro medio}

Las pruebas de denominación disponibles actualmente en Argentina no contemplan en su totalidad los aspectos recién mencionados. Por ejemplo, una de las más utilizadas en la práctica clínica, el Test de Denominación de Boston (TDB de Kaplan, Goodglass \& Weintraub, 1983) no cuenta con el control de las variables que afectan los procesos previos al acceso al léxico (nivel de análisis visual, recuperación de la representación estructural de los objetos y el acceso conceptual-semántico) (Manoiloff, 2010). A su vez, no ha sido creada originalmente para población argentina, por lo cual no tiene en cuenta las variables sociales y culturales. $\mathrm{La}$ adaptación del TDB realizada por Allegri y colaboradores (1997) si bien reorganizó la presentación de las láminas en función de la dificultad observada en una muestra local, no incluyó el control de variables psicolingüísticas mediante normas regionales al momento de la elección y el ordenamiento de las imágenes. Del mismo modo, la elección de las claves semánticas no se basa en estudios normativos locales, que permiten conocer los rasgos distintivos que describen al concepto que representa el objeto en nuestra población. Como consecuencia de esto, se ha observado reiteradamente durante la administración del TDB que muchas de las imágenes tienen respuestas relacionadas a otros objetos culturalmente significativos en lugar de la respuesta establecida como correcta. Por ejemplo, la imagen del iglu, fue varias veces confundida con un horno de barro u horno de pan; el transportador, en personas oriundas del Noroeste Argentino es conocido como Semicírculo; no pudiendo 
ser denominado por el nombre que provee el manual, por lo que se llega al interrogante si considerar incorrecta o no una respuesta de algo que no se conoce bajo ese nombre.

Otra de las pruebas de denominación que ha obtenido evidencias de validez en Argentina es el Test de Oldfield y Wingfield (1965). Un aspecto interesante de la validación realizada sobre este test es que se utilizó población clínica, tanto con lesiones neurológicas focales como difusas (Vigliecca, Aleman \& Jaime, 2007). Sin embargo, no cuenta con facilitadores fonológicos y semánticos, punto importante, ya que las fallas o aciertos con su uso, permiten inferir información relevante sobre el perfil cognitivo, como se señaló anteriormente.

También se ha desarrollado a nivel local el Test Córdoba de Denominación (Fernández, 2013) que busca incluir estímulos de variada frecuencia de uso. Los datos normativos fueron extraídos de las normas españolas disponibles en ese momento y los dibujos especialmente generados para la tarea. En su construcción no se ha controlado la complejidad visual ni acuerdo en la imagen de los dibujos, presentando dibujos muy simples y otros muy complejos, incluso varios de ellos con problemas de reconocimiento (generando muchos errores visuales, por ejemplo el ítem microscopio es frecuentemente reconocido como prensa, y delfín como ballena). Además, presenta mayor cantidad de objetos que son de conocimiento prioritariamente masculino (como armas y herramientas). A su vez, muchas de las claves semánticas no funcionan como facilitadores. Por ejemplo para el ítem cobra, los sujetos dan como primera respuesta espontánea víbora y la clave semántica consiste en es un animal, que no aportaría datos adicionales para el reconocimiento del concepto que representa el dibujo, ni la etiqueta lexical con que se designa.

La Prueba Argentina Psicolingüística de Denominación de Imágenes (PAPDI) presenta algunas cualidades que la diferencian de las recién mencionadas en la medida en que se contemplaron los siguientes aspectos para su construcción: (a) los estímulos pic- tóricos fueron seleccionados en base a un set de amplio uso en Psicología Experimental; (b) se controlaron las variables psicolingüísticas de las imágenes y de sus nombres (léxico) en base a normas locales; (c) se incluyeron claves fonológicas y semánticas; (d) las claves semánticas fueron también elaboradas tomando como referencia normas locales y (e) es lo suficientemente breve para su uso en la clínica y de libre acceso.

\section{La Prueba Argentina Psicolingüística de Denominación de Imágenes (PAPDI). Su construcción}

\section{Metodología}

Las imágenes que conforman la prueba fueron extraídas del set de 400 imágenes experimentales publicadas por Cycowicz, Friedman, Rothstein, y Snodgrass (1997), que incluye el conjunto publicado anteriormente por Snodgrass y Vanderwart (1980). Se seleccionó este set porque es de amplio uso en Psicología Experimental y Neuropsicología (por ejemplo, Abutalebi et al., 2008; Bates et al., 2003; Fernández, Diez, Alonso $\&$ Beato, 2004). Se cuenta con normas psicolingüísticas argentinas para estas imágenes (Manoiloff et al., 2010) lo cual permitió seleccionar las imágenes controlando y manipulando los efectos de las variables que afectan las diferentes etapas de procesamiento.

En primer lugar se buscó seleccionar un pool de imágenes controlando las características visuales de los dibujos. Esto consistió en considerar dibujos que tengan un valor medio a bajo en el índice de Complejidad Visual (en cantidad de líneas y detalles), es decir que tengan valores menores a 3.14 según el trabajo de Manoiloff y colaboradores (2010). Los mismos debían tener un grado medio a alto de concordancia entre la imagen mental del objeto y su correspondiente representación pictórica (valores mayores a 3.53 en Concordancia con la Imagen según Manoiloff et al., 2010). Este control permite asegurar que no haya diferencias importantes en el análisis visual del dibujo ni en la facilidad del reconocimiento del objeto para los 
ítems seleccionados. Si hay fallas en la respuesta, este emparejamiento disminuye las probabilidades de que se deba a problemas en las etapas iniciales de procesamiento de la imagen. Además, los estímulos debían ser medianamente familiares (con valores mayores a 2.81 según Manoiloff et al., 2010) y poseer una variabilidad de la imagen de media a alta (es decir, tener un valor mayor a 1.15 , según Manoiloff y colaboradores, 2010, en el número de diferentes figuras que son evocadas por el nombre del objeto). Con este control se evita tener conceptos muy diferentes en cuanto a su procesamiento semántico, ya que tanto aquellos con alta familiaridad como aquellos con mucha variabilidad en la imagen facilitan el procesamiento. Asimismo, estas imágenes debían tener un porcentaje de acuerdo en el nombre arriba del $80 \%$ (según Manoiloff et al., 2010) para utilizar imágenes que sean nombradas mayoritariamente con una sola palabra, y no tener múltiples designaciones, ya que esto demora $\mathrm{y}$ dificulta la selección de una etiqueta lexical así como la corrección (Alario \& Ferrand, 1999). Para este índice es de suma relevancia contar con estudios normativos locales, ya que ha demostrado ser muy dependiente del idioma y / o cultura de donde se obtiene (Alario \& Ferrand, 1999; De11'Acqua et al., 2000; Martínez-Cuitiño et al., 2015; Sanfeliu \& Fernández, 1996). En base a estos criterios fueron seleccionadas 62 imágenes del set de 400 .

Un punto importante en la construcción de este tipo de pruebas es manipular las variables que afectan la etapa de recuperación de la forma fonológica del nombre (o etiqueta lexical) para generar un gradiente de dificultad en los ítems de la prueba. Con esta finalidad se consideraron las variables de Frecuencia léxica y Edad de adquisición. La primera hace referencia a la frecuencia de uso de una palabra en una lengua dada. $\mathrm{Su}$ valor puede ser obtenido de forma objetiva (aparición de muestras de material escrito $\mathrm{u}$ oral en grandes corpus), o de forma subjetiva (valoración de cada hablante del uso que realiza de esa palabra). Este índice permite entonces ordenar las palabras en términos de mayor o menor frecuencia de uso. El clásico efecto de frecuencia (EF), demostrado por primera vez por Oldfield y Wingfield en 1965, se traduce en el hecho de que los objetos cuyos nombres son frecuentes en la lengua son denominados más rápido y acertadamente que aquellos de nombre menos frecuente (y esto fue confirmado posteriormente por varias investigaciones como las de Humphreys et al., 1988; Jescheniak \& Levelt, 1994; Levelt et al., 1999).

Al momento de la creación de la prueba no se contaba con un diccionario de frecuencia de uso construido en nuestro contexto (posteriormente fueron publicadas las normas de Martínez-Cuitiño et al., 2015 que obtuvieron la frecuencia subjetiva para el set experimental de Cycowicz et al. en la Ciudad Autónoma de Buenos Aires). Por lo tanto, el segundo paso, fue obtener un índice de frecuencia subjetiva de uso del nombre para el pool de las 62 imágenes seleccionadas. También, como medida objetiva de frecuencia de uso, se consideró la frecuencia de aparición del nombre arrojada por el LEXESP (Sebastián-Gallés, Martí, Cuetos \& Carreiras, 2000) obtenida en España. No obstante, es importante mencionar que estudios previos muestran que la estimación subjetiva es un mejor predictor del procesamiento léxico que las normas de frecuencias objetivas (Balota, Pilotti \& Cortese, 2001; Meschyan \& Hernández, 2002; Manoiloff, Seguí \& Ha1lé, 2013). Para la estimación de la frecuencia subjetiva se utilizó a sujetos de nuestro medio (Córdoba Capital), con edades de 20 a 70 años y con diferentes niveles educativos. Y se obtuvo la media de las estimaciones para cada palabra.

Para conformar la primera versión de la prueba, las 62 imágenes seleccionadas fueron ordenadas de acuerdo a la variable Edad de Adquisición. Varios trabajos han encontrado que los objetos cuyos nombres fueron aprendidos tempranamente son denominados más rápida y acertadamente que aquellos aprendidos tardíamente. Además, esta variable mostró estar fuertemente relacionada con la variable de Frecuencia de Uso (Alario et al., 2004; Barry et al., 1997; Ellis \& Morrison, 
1998; Morrison \& Ellis, 1995; Snodgrass \& Yuditsky, 1996). Por ello se decidió utilizar la Edad de Adquisición como primer criterio de orden. Siguiendo lo propuesto por Márquez de la Plata y sus colaboradores (2008), los nombres de las figuras seleccionadas fueron ubicados en una escala de acuerdo al puntaje $\mathrm{z}$ con respecto a la media general de la variable $(M=2.52, D E=.79$, según Manoiloff et al., 2010), para crear tres grupos subsecuentes: uno conformado por todos los nombres que tuvieran una desviación estándar por arriba de la media; otro con una desviación estándar por debajo y un tercero constituido por los nombres que tenían valores intermedios. La frecuencia subjetiva de uso fue utilizada para ordenar los nombres de las imágenes dentro de cada grupo conformado con la variable Edad de adquisición. Las palabras con más alta frecuencia de uso fueron ubicadas primero. Cuando dos palabras compartían el mismo valor de frecuencia subjetiva, se las ordenó según su frecuencia objetiva obtenida del LEXESP.

Una vez que las 62 imágenes fueron seleccionadas y ordenadas se construyeron sus respectivas claves semánticas y fonológicas. Las claves semánticas fueron extraídas de las Normas de Producción de Atributos Semánticos en español (Vivas, J., Vivas, L., Comesaña, García Coni \& Vorano, 2016) recogidas con muestra argentina. Se seleccionaron aquellos atributos de mayor relevancia para cada concepto. En los casos en que había una categoría semántica clara de pertenencia también se agregó esa información (por ejemplo, es un electrodoméstico -categoría supraordinaria- que sirve para conservar alimentos en buen estado -descripción más relevante del concepto según Vivas et al., 2016). Sólo se seleccionó el segundo atributo de mayor relevancia en aquellos casos en los cuales el primer atributo podía ser considerado un facilitador fonológico (por ejemplo, para el concepto botón el atributo sirve para abotonar). Por su parte, las claves fonológicas correspondieron a la primera sílaba de cada nombre (por ejemplo, para el ítem botón, la sílaba bo).

\section{Prueba piloto y selección de estímulos}

El set de 62 figuras fue administrado a un grupo de 50 personas de distintas edades, ambos sexos y tres niveles educativos (primario, secundario y terciario). En base a las respuestas obtenidas, se eliminaron 32 figuras siguiendo los criterios utilizados en el desarrollo de pruebas similares (Fernández, 2013):

1.- figuras que no evocaban una respuesta unívoca, es decir que fueran fácilmente confundibles con otros objetos,

2.- figuras que tuvieran más de una respuesta aceptable aunque no todas fuesen correctas y

3.- ítems que presentaran efecto techo donde las puntuaciones se acumularan en puntajes altos y no permitieran discriminar la capacidad de los participantes para seleccionar, recuperar y producir una palabra. En la constitución de estas pruebas, habitualmente se incluyen entre 25 y $30 \%$ de los ítems con dicha característica, pues la prueba debe ir incorporando ítems con dificultad creciente para lograr el objetivo de evaluación.

A su vez, se utilizaron criterios adicionales para la selección de las imágenes definitivas:

a.- se removieron las imágenes que fueran compartidas entre los distintos tests de denominación más utilizadas como el Test de Boston. Por ejemplo, Cama, Lápiz, Tijera, Peine, Escoba, Aguja, Hamaca, Percha, Helicóptero, para obtener una prueba con imágenes novedosas y no afectar a la prueba de validez convergente.

b.- Se suprimieron imágenes que en la prueba piloto no recibieron respuestas unívocas (pan, libro, bandera).

En base a estos criterios se removieron 42 ítems (Cama, Mesa, Vaso, Perro, Cuchara, Papa, Pierna, Oreja, Luna, Libro, Botella, Pantalón, Puerta, Silla, Taza, Queso, Brazo, Gato, Camisa, Lápiz, Cebolla, Banana, Tijera, Corazón, Limón, Peine, Escoba, Copa, Aguja, Vela, Pera, Regla, Bandera, Hamaca, Cigarrillo, Cenicero, Percha, Helicóptero, Destornillador, Tornillo, Martillo, Corbata) y se incluyeron 10 ítems adicionales (Broche, 
Cadena, Chaleco, Barrilete, Hacha, Pandereta, Yo-yo, Ancla, Dedal, Anzuelo). Nuevamente, se volvieron a ordenar los ítems siguiendo los valores de Edad de Adquisición y la frecuencia subjetiva de uso (para los ítems nuevos) y su dificultad en la denominación (para los ítems viejos), es decir, siguiendo el porcentaje de aciertos (o errores) en la respuesta espontánea (sin la presentación de ninguna clave). La versión final puede verse en el Anexo.

Es importante mencionar para el uso de la PAPDI a nivel nacional, que el trabajo de nuevas normas léxicas y semánticas para el set de 400 figuras de Cycowicz y colaboradores (1997) desarrollado por Martínez-Cuitiño y colaboradores (2015) en la ciudad de Buenos Aires, en comparación con el trabajo de Manoiloff y colaboradores (2010) desarrollado en Córdoba, no presenta diferencias significativas en la variable Acuerdo en el nombre para las imágenes incluidas en la prueba.

\section{Conclusión}

En esta primera parte del trabajo se presenta la construcción y desarrollo de una prueba de denominación de imágenes:
PAPDI. Para ello, primero se exhibieron las características particulares del proceso que se lleva a cabo para poder nombrar una figura y las variables que afectan dicho proceso. En el proceso de construcción y desarrollo de la prueba se muestra cómo se tuvieron en cuenta estas particularidades y cuáles variables han sido controladas y cuáles manipuladas. La obtención de los índices que representan estas variables son dependientes del contexto cultural y lingüístico en los cuales fueron desarrollados lo cual hace esencial el uso de normas locales. Esta influencia es más marcada para las variables que fueron manipuladas para la construcción de la prueba: Frecuencia léxica y Edad de adquisición; ya que las mismas afectan una etapa fundamental que busca evaluar la tarea de denominación de imágenes, la activación, acceso y recuperación de la forma fonológica del nombre (etiqueta lexical). Además, se tuvo en cuenta el valor de las claves semánticas y fonológicas, tanto en su construcción como en su uso, y nuevamente, la importancia de contar con normas locales para la elaboración de las claves semánticas.

En la segunda parte del trabajo se presentarán los estudios psicométricos que otorgan evidencia de su pertinencia y validez.

\section{Anexo}

Versión final de la Prueba Argentina Psicolingüistica de Denominación de Imágenes

\begin{tabular}{|c|c|c|c|c|c|c|}
\hline \multirow{2}{*}{ IMÁGENES } & \multirow{2}{*}{$\begin{array}{l}\text { Correcta } \\
\mathrm{S} / \mathrm{C}\end{array}$} & \multicolumn{2}{|c|}{$\begin{array}{l}\text { Clave } \\
\text { semántica }\end{array}$} & \multicolumn{2}{|c|}{$\begin{array}{c}\text { Clave } \\
\text { fonológica }\end{array}$} & \multirow{2}{*}{ Respuestas } \\
\hline & & $\mathrm{C}$ & I & $\mathrm{C}$ & I & \\
\hline $\begin{array}{l}\text { 1. Heladera (electrodoméstico que } \\
\text { sirve para conservar alimentos } \\
\text { en buen estado) }\end{array}$ & & & & & & \\
\hline $\begin{array}{l}\text { 2. Tenedor (utensilio que sirve } \\
\text { para comer) }\end{array}$ & & & & & & \\
\hline $\begin{array}{l}\text { 3. Broche (elemento que sirve para } \\
\text { colgar prendas de vestir) }\end{array}$ & & & & & & \\
\hline $\begin{array}{l}\text { 4. Cadena (Objeto que tiene } \\
\text { eslabones, que se usaba para atar } \\
\text { a los presos) }\end{array}$ & & & & & & \\
\hline $\begin{array}{l}\text { 5- } \underline{\text { Jarra (recipiente que se usa para }} \\
\text { servir líquidos) }\end{array}$ & & & & & & \\
\hline 6. Estrella (astro brillante) & & & & & & \\
\hline
\end{tabular}

(Continúa) 


\section{Anexo (Continuación)}

Versión final de la Prueba Argentina Psicolingüística de Denominación de Imágenes

\begin{tabular}{|c|c|c|c|c|c|c|}
\hline \multirow{2}{*}{ IMÁGENES } & \multirow{2}{*}{$\begin{array}{l}\text { Correcta } \\
\mathrm{S} / \mathrm{C}\end{array}$} & \multicolumn{2}{|c|}{$\begin{array}{c}\text { Clave } \\
\text { semántica }\end{array}$} & \multicolumn{2}{|c|}{$\begin{array}{l}\text { Clave } \\
\text { fonológica }\end{array}$} & \multirow{2}{*}{ Respuestas } \\
\hline & & $\mathrm{C}$ & $\mathrm{I}$ & $\mathrm{C}$ & $\mathrm{I}$ & \\
\hline \multicolumn{7}{|l|}{$\begin{array}{l}\text { 7. Chaleco (prenda de vestir sin } \\
\text { mangas) }\end{array}$} \\
\hline \multicolumn{7}{|l|}{$\begin{array}{l}\text { 8. Sobre (sirve para guardar } \\
\text { papeles) }\end{array}$} \\
\hline \multicolumn{7}{|l|}{$\begin{array}{l}\text { 9. Moño (adorno que se usa en los } \\
\text { regalos) }\end{array}$} \\
\hline \multicolumn{7}{|l|}{ 10. Sartén (sirve para freír) } \\
\hline \multicolumn{7}{|l|}{$\begin{array}{l}\text { 11. Murciélago (animal nocturno } \\
\text { que vuela) }\end{array}$} \\
\hline \multicolumn{7}{|l|}{$\begin{array}{l}\text { 12. Barrilete (juguete de papel que } \\
\text { lo usan con el viento) }\end{array}$} \\
\hline \multicolumn{7}{|l|}{$\begin{array}{l}\text { 13. Media (prenda de vestir que se } \\
\text { usa en los pies) }\end{array}$} \\
\hline \multicolumn{7}{|l|}{$\begin{array}{l}\text { 14. Tomate (verdura que se usa } \\
\text { para ensalada) }\end{array}$} \\
\hline \multicolumn{7}{|l|}{$\begin{array}{l}\text { 15. Manzana (fruta de cáscara } \\
\text { roja) }\end{array}$} \\
\hline \multicolumn{7}{|l|}{$\begin{array}{l}\text { 16. Botón (sirve para cerrar } \\
\text { prendas de vestir) }\end{array}$} \\
\hline \multicolumn{7}{|l|}{$\begin{array}{l}\text { 17. Pecera (contiene agua y } \\
\text { animales acuáticos) }\end{array}$} \\
\hline \multicolumn{7}{|l|}{$\begin{array}{l}\text { 18. Clavo (se usa con un martillo, } \\
\text { sirve para colgar) }\end{array}$} \\
\hline \multicolumn{7}{|l|}{$\begin{array}{l}\text { 19. Cisne (ave blanca que vive en } \\
\text { el lago) }\end{array}$} \\
\hline \multicolumn{7}{|l|}{ 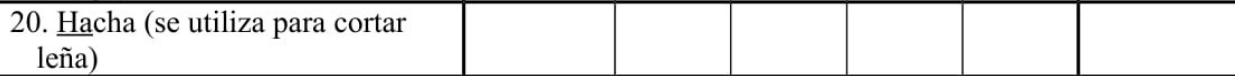 } \\
\hline \multicolumn{7}{|l|}{$\begin{array}{l}\text { 21. Pandereta (es un instrumento } \\
\text { musical de percusión) }\end{array}$} \\
\hline \multicolumn{7}{|l|}{$\begin{array}{l}\text { 22. Anillo (representa } \\
\text { compromiso) }\end{array}$} \\
\hline \multicolumn{7}{|l|}{$\begin{array}{l}\text { 23. Yo-Yo (es un juguete que tiene } \\
\text { un cordel) }\end{array}$} \\
\hline \multicolumn{7}{|l|}{$\begin{array}{l}\text { 24. Ancla (sirve para mantener } \\
\text { barcos en un lugar fijo) }\end{array}$} \\
\hline \multicolumn{7}{|l|}{$\begin{array}{l}\text { 25. Nariz (parte de la cara que } \\
\text { sirve para respirar) }\end{array}$} \\
\hline \multicolumn{7}{|l|}{ 26. Trompo (juguete que gira) } \\
\hline \multicolumn{7}{|l|}{ 27. Lata (sirve para conservar) } \\
\hline \multicolumn{7}{|l|}{$\begin{array}{l}\text { 28. Dedal (se usa en el dedo y } \\
\text { sirve para coser) }\end{array}$} \\
\hline \multicolumn{7}{|l|}{$\begin{array}{l}\text { 29. Hamburguesa (comida hecha } \\
\text { con pan y carne) }\end{array}$} \\
\hline 30. Anzuelo (sirve para pescar) & & & & & & \\
\hline
\end{tabular}




\section{Referencias bibliográficas}

Abutalebi, J., Annoni, J.M., Zimine, I., Pegna, A.J., Seghier, M.L. et al. (2008). Language control and lexical competition in bilinguals: An event-related FMRI study. Cerebral Cortex, 18, 1496-505. http://dx. doi.org/10.1093/cercor/bhm 182

Alario, F-X. \& Ferrand, L. (1999). A set of 400 pictures standardized for French: Norms for name agreement, image agreement, familiarity, visual complexity, image variability and age of acquisition. Behavior Research Methods, Instruments \& Computers, 31(3), 531- 552. http://dx.doi.org/10.3758/BF032 00732

Alario, F.-X., Ferrand, L., Laganaro, M., New, B., Frauenfelder, U. H.\& Seguí, J. (2004). Predictors of picture naming speed. Behavior Research Methods, Instruments, \& Computers, 36, 140-155. http://dx.doi.org/ 10.3758/BF03195559

Allegri, R., Mangone, C., Rymberg, S., Taragano, F. \& Baumann, D. (1997). Spanish Boston Naming Test Norms. The Clinical Neuropsychologist, 11(4), 416-420. http://dx.doi.org/10.1080/138540497084004 71

Balota, D. A., Pilotti, M. \& Cortese, M. J. (2001). Subjective frequency estimates for 2,938 monosyllabic words. Memory \& Cognition, 29, 639-647. http://dx.doi.org/ 10.3758/BF03200465

Barbón, A. \& Cuetos, F. (2006). Efectos de la edad de adquisición en tareas de categorización semántica [Age of aquisition effects in semantic categorization tasks]. Psicológica, 27, 207-223.

Barry, C., Morrison, C. \& Ellis, A.W. (1997). Naming the Snodgrass and Vanderwart pictures: Effects of age of acquisition, frequency and name agreement. Quarterly Journal of Experimental Psychology, 50 A, 560-585. http://dx.doi.org/10.1080/ 027249 897392026
Bates, E., D’Amico, S., Jacobsen, T., Székely, A., Andonova, E., Devescovi, A. et al. (2003). Timed picture naming in seven languages. Psychonomic Bulletin Review, 10(2), 344-380. http://dx.doi.org/10.3758/ BF03196494

Bonin, P., Boyer, B., Méot, A., Fayol, M. \& Droit, S. (2004). Psycholinguistic norms for action photographs in French and their relationships with spoken and written latencies. Behavior Research Methods, Instruments, \& Computers, 36, 127-139. http://dx.doi.org/ 10.3758/BF03195558

Corbett, F., Jefferies, E., Burns, A. \& Lambon Ralph, M. (2012). Unpicking the semantic impairment in Alzheimer's disease: Qualitative changes with disease severity. Behavioural Neurology, 25, 23-34. http:// dx.doi.org/10.3233/BEN-2012-0346.

Cuetos, F., Ellis, A.W. \& Alvarez, B. (1999). Naming times for the Snodgrass and Vanderwart pictures in Spanish. Behavior Research Methods, Instruments, \& Computers, 31(4), 650-658. http://dx.doi. org/10.3758/BF03200741

Cuetos, F., González, J. \& de Vega, M. (2015). Psicología del lenguaje [Language Psychology]. Madrid: Editorial Médica Paname-ricana.

Cycowicz, Y.M., Friedman, D., Rothstein, M. \& Snodgrass, J.G. (1997). Picture naming by young children: norms for name agreement, familiarity, and visual complexity. Journal of Experimental Child Psychology, 65(2), 171-237. http://dx.doi.org/10.1006/ jecp.1996.2356

Dell'Acqua, R., Lotto, L. \& Job, R. (2000). Naming times and standardized norms for the Italian PD/DPSS set of 266 pictures: Direct comparisons with American, English, French, and Spanish published databases. Behavior Research Methods, Instruments, \& Computers, 32, 588-615. http://dx.doi.org/ 10.3758/BF03200832

Ellis, A.W. \& Morrison, C.M. (1998). Real ageof-acquisition effects in lexical retrieval. 
Journal of Experimental Psychology: Learning, Memory, and Cognition, 24(2), 515-523. http://dx.doi.org/10.1037/02787393.24.2.515

Fernández, A. (2013). Development of a Confrontation Naming Test For Spanishspeakers: The Cordoba Naming Test. The Clinical Neuropsychologist, 27(7), 11791198. http://dx.doi.org/10.1080/13854046. 2013.822931

Fernández, A., Díez, E., Alonso, M.A. \& Beato, M.S. (2004). Free Association Norms for the Spanish Names of the Snodgrass and Vanderwart Pictures. Behavior Research Methods, Instruments \& Computers, 36(34), 577-584. http://dx.doi.org/10.3758/BF0 3195604

Glaser, WR. (1992). Picture naming. Cognition, 42(1), 61-105.

Grasso, L. \& Peraita, H. (2011). Adaptación de la Batería de Evaluación de la Memoria Semántica en la Demencia de tipo Alzheimer (EMSDA) a la población de la ciudad de Buenos Aires [Evaluation battery for Semantic Memory Deterioration in Dementia of the Alzheimer type (EMSDA): Item's adjustment to the population of Buenos Aires city]. Interdisciplinaria, 28(1), 37-56.

Humphreys, G.W., Riddoch, M.J. \& Quinlan, P.T. (1988). Cascade processes in picture identification. Cognitive Neuropsychology, 5, 67-103.

Iyer, G., Saccuman, C., Bates, E. \& Wulfeck, B. (2001). A study of age-of-acquisition (AoA) ratings in adults. Center for Research in Language, 13(2). Recuperado el 1 de marzo de 2017 de ftp://ftp.crl.ucsd.edu/ pub/newsletter/pdf/13-2.pdf

Jefferies, E., Baker, S.S., Doran, M. \& Ralph, M.A.L. (2007). Refractory effects in stroke aphasia: A consequence of poor semantic control. Neuropsychologia, 45(5), 10651079. http://dx.doi.org/10.1016/j.neuropsychologia.2006.09.009
Jefferies, E., Patterson, K. \& Lambon Ralph, M.A. (2008). Deficits of knowledge versus control in semantic cognition: insight from cued naming. Neuropsychologia, 46, 649658. http://dx.doi.org/10.1016/j.neuropsychologia.2007.09.007

Jescheniak, J.D. \& Levelt, W.J.M. (1994). Word frequency effects in speech production: Retrieval of syntactic information and of phonological form. Journal of Experimental Psychology: Learning, Memory, and Cognition, 20, 824-843.

Kaplan, E., Goodglass, H. \& Weintraub, S. (1983). Boston naming test. Philadelphia: Lea \& Febiger.

Levelt, W.J.M., Roelofs, A. \& Meyer, A.S. (1999). A theory of lexical access in speech production. The Behavioral and Brain Sciences, 22, 1-38. disc. 38-75.

Lezak, M.D. (1976). Neuropsychological Assessment. Oxford, England: Oxford University Press.

Manoiloff, L. (2010). El acceso al léxico a partir de un objeto: Propiedades fonológicas y ortográficas [The phonological access from an object: Phonological and ortographic properties]. Tesis doctoral no publicada. Facultad de Psicología. Universidad Nacional de Córdoba. Argentina.

Manoiloff, L., Arstein, M., Canavoso Fernández, L. \& Segui, J. (2010). Expanded Norms for 400 Experimental Pictures in an Argentinian Spanish-Speaking Population. Behavior Research Methods, 42(2), 452460. http://dx.doi.org/10.3758/BRM.42.2. 452

Manoiloff, L., Segui, J. \& Hallé, P. (2013). L'effet de fréquence dans l'accès aux propriétés phonologiques des noms d'objets. L'Année psychologique, 113(03), 335-348. http://dx.doi.org/10.4074/S0003503313003 023

Marquez de la Plata, M., Vicioso, B., Hynan, L., Evans, H.M., Diaz-Arrastia, R., Lacritz, L. \& Cullum, C.M. (2007). Development of 
the Texas Spanish Naming Test: A test for Spanish speakers. The Clinical Neuropsychologist, 22, 288-304. http://dx.doi.org/ 10.1080/13854040701250470

Martínez-Cuitiño, M., Barreyro, J.P., Wilson, M. \& Jaichenco, V. (2015). Nuevas normas semánticas y de tiempos de latencia para un set de 400 dibujos en español. [New norms semantic and latency time for a set of 400 drawings in Spanish]. Interdisciplinaria, 32(2), 289-305. Recuperado el 1 de marzo de 2017 de http://www.scielo.org.ar/scielo. php? script $=$ sci_arttext $\&$ pid $=$ S 1668 $70272015000200006 \& \operatorname{lng}=\mathrm{es} \& \ln \mathrm{ln}=\mathrm{es}$.

Martínez-Cuitiño, M. \& Jaichenco, V. (2012). Evaluación de la memoria semántica. [Semantic memory assessment]. Revista de Psicología, 8(16). Recuperado e1 24 de agosto de 2016 de http://bibliotecadigital. uca.edu.ar/repositorio/revistas/evaluacionmemoria-semantica-cuitino-jaichenco.pdf

Mehri, A., Jahani, Y., Alemi, R. \& Aramipour, E. (2008). Investigation of usage of semantic and phonological cues in naming of fluent aphasia and Alzheimer Disease. Audiology and Vestibular Research, 16(2), 51-56.

Meschyan, G. \& Hernandez, A. (2002). Age of acquisition and word frequency: Determinants of object-naming speed and accuracy. Memory \& Cognition, 30(2), 262269. http://dx.doi.org/10.3758/BF03195287

Moreno Martínez, F.J. (2006). Una revisión de las principales tareas para evaluar el deterioro semántico en la enfermedad de Alzheimer [A review of the main tasks used to assess the semantic impairment in Alzheimer's Disease]. Acción Psicológica, 4(1), 57-68.

Morrison, C.M., Chappell, T.D. \& Ellis, A.W. (1997). Age of acquisition norms for a large set of object names and their relation to adult estimates and other variables. Quarterly Journal of Experimental Psychol- ogy, 50A, 528-559. http://dx.doi.org/10. 1080/027249897392017

Morrison, C.M. \& Ellis, A.W. (1995). Roles of word frequency and age of acquisition in word naming and lexical decision. Journal of Experimental Psychology: Learning, Memory, and Cognition, 21, 116-133. http:// dx.doi.org/10.1037/0278-7393.21.1.116

Oldfield, R.C. \& Wingfield, A. (1965). Response latencies in naming objects. Quartery Journal of Experimental Psychology, 17, 273-81. http://dx.doi.org/10.1080/ 17470216508416445

Sanfeliu, M.C. \& Fernández, A. (1996). A set of 254 Snodgrass-Vanderwart pictures standardized for Spanish: Norms for name agreement, image agreement, familiarity and visual complexity. Behavior Research Methods, Instrument \& Computers, 28(4), 537-555. http://dx.doi.org/10.3758/BF032 00541

Sebastián-Gallés, N., Martí, M.A., Cuetos, F. \& Carreiras, M. (2000). LEXESP: Una base de datos informatizada del español. [LEXESP: an informatized data base of Spanish]. Barcelona: Universitat de Barcelona.

Snodgrass, J.G. \& Vanderwart, M. (1980). A standardized set of 260 pictures: Norms for name agreement, image agreement, familiarity, and visual complexity. Journal of Experimental Psychology: Human Learning \& Memory, 6(2), 174-215. http://dx.doi.org/ 10.1037/0278-7393.6.2.174

Snodgrass, J. G. \& Yuditsky, T. (1996). Naming times for the Snodgrass and Vanderwart pictures. Behavior Research Methods, Instruments, \& Computers, 28, 516-536. http://dx.doi.org/10.3758/BF03200540

Vigliecca, N.S., Aleman, G.P. \& Jaime, M.P. (2007). Adaptación y validación de un test de denominación en hispanohablantes: fiabilidad y discriminación de pacientes con demencia y con lesiones cerebrales unilaterales [Adaptation and validation of a naming 
test in Spanish speakers: reliability and discrimination of patients with dementia and unilateral brain lesions]. Neurología, 22, 147-152.

Vivas, J., Vivas, L., Comesaña, A., García Coni, A. \& Vorano, A. (2017). Spanish semantic feature production norms for 400 concrete concepts. Behavior Research Methods, 49(3), 1095-1106. 